\title{
AIS-enabled collision avoidance strategies for autonomous sailboats
}

\author{
José C. Alves and Nuno A. Cruz
}

\begin{abstract}
Autonomous sailing boats are a valuable resource for ocean monitoring, sampling and surveillance due to their intrinsic very low energy requirements and extreme capability for long term navigation. However, the extended presence in the ocean is vulnerable to a multitude of hazards that may compromise the success of a mission. Although the conventional manned sea crafts must comply to the international rules of the road (or COLREGS), unmanned (small and slow) robotic boats should actively anticipate close encounters with (large and fast) ships that in practical situations may not be able to avoid collisions with small vessels. This paper proposes a set of simple rules for implementing a defensive and conservative collision avoidance strategy for autonomous sailing boats, based solely on the AIS data received from large ships.
\end{abstract}

\section{Introduction}

Unmanned autonomous sailing boats are a proven technology for ocean monitoring, sampling and surveillance due to their intrinsic very low energy requirements, good navigation ability and extreme potential for long navigation journeys [9]. However, maintaining an operation in the sea surface for long periods of time is susceptible to various risks of failure due to external threats. Environmental conditions can now be predicted for a few days window with high degrees of confidence, thus allowing re-plan a mission in advance to avoid potential dangerous wind and sea states. However, the risk of collision with other vessels or floating debris represents a sig-

José C. Alves

FEUP/INESC TEC, Rua Dr Roberto Frias 4200-465 PORTO, Portugal, e-mail: jca@ fe.up.pt

Nuno A. Cruz

FEUP/INESC TEC, Rua Dr Roberto Frias 4200-465 PORTO, Portugal, e-mail: nacruz@ fe.up.pt 
nificant menace especially when operating in regions of intense marine traffic, like the English Channel or the west coast of Portugal and Spain.

The detection of small boats, floating fishing nets or partially submerged wrecks (e.g. lost containers) requires onboard active devices like radar, image or acoustic sensors and the effective detection range depends significantly on the size of the boat (more precisely the height of the sensors), the state of the sea and, for underwater imaging, the transparency of the water. Although the avoidance of collisions with such small objects may be effectively done with last minute maneuvers executed at a short distance of the object (in a practical range of tens of meters), the situation with large ships must be handled in a very different manner in order to ensure a comfortable distance of safety, that depends on the size of the meeting ships and hence on their relative maneuverability. This is a general principle clearly stated in the COLREGS [4] and adopted by captains of real ships: for example, if $1 \mathrm{~m}$ may be a safe minimum distance between two small sailing dinghies, a $100 \mathrm{~m}$ separation between two large tankers sailing at cruising speed is certainly considered a severe near miss. Thus, an effective mechanism for avoiding collisions with large ships ${ }^{1}$ must be able to guarantee the appropriate means for executing a convenient and timely maneuver to improve the safety margin.

Although all ships must obey to the international collision avoidance regulations (COLREGS), when ships meet in a potential collision route the decision to maneuver for avoiding the collision may be dictated by other factors than the simple right of way stated by the rules. For example, both ships must be aware of the speed, course and approximate position of each other, and the relative maneuverability of the meeting ships must be taken into account. Considering situations involving the encounter between a small (and slow) robotic vessel (ASV) and a large (and fast) ship, some realistic assumptions need to be taken into consideration:

- Large ships navigate most of the time under autopilot and human surveillance. A small and possibly intermittent radar echo may be ignored if it is not visually confirmed by the crew.

- A large ship will only have clear visual contact with a small ASV within a few hundred meters range (by night the navigation lights may improve the visibility). This may be too close for effectively maneuvering to avoid a collision.

- Small ASVs, and specially robotic sailing vessels, may not be able to carry or generate enough energy to power a AIS (Automatic Identification System) transponder, so they will not be detected at large distances from other vessels.

If the robotic vessel is a sailing boat, several additional constraints must be considered for implementing collision avoidance maneuvers, in spite of the default rule stated in COLREGS giving the right-of-way to sailing ships. A sailing boat cannot naturally navigate outside of the feasible wind angles, cannot increase its speed (usually sailing boats always seek its maximum speed) and in certain situations a sailing boat may also not be able to reduce its speed, as for example in a dead downwind course with certain types of rigs.

\footnotetext{
${ }^{1}$ The term large must be understood in relation to the size of the autonomous boat
} 
Presently the situational picture of the surrounding (large) vessels can be easily done by decoding the AIS (Automatic Identification System) messages transmitted in the maritime VHF band. Although the transmission of AIS data is not yet compulsory for all ships, presently all commercial ships and fishing vessels longer than $16 \mathrm{~m}$ must broadcast their navigation status using the AIS. With the continuous reduction of the cost of transponders, many pleasure vessels also opt to use AIS as an additional safety asset and in the next years it is expected that more and more boats will be equipped with AIS. Even though to transmit AIS data it is required a certified equipment, an operating license and a unique ship identification number (MMSI - Maritime Mobile Service Identity), the reception of AIS data only needs a dedicated AIS receiver or a radio receiver for the AIS radio frequencies and software to decode, extract and interpret the AIS data.

To minimize the risk of collision with other ships, a (small) robotic sailing craft can adopt a defensive behavior to avoid possible near miss situations and anticipate maneuvers for reducing the risk of collision. In this paper we propose a defensive navigation strategy for collision avoidance, based on the AIS received data or by any other means capable of providing quasi-real-time the position, course and speed of other vessels. Instead to comply to the COLREGS, which in most situations gives the right-of-way to sailing boats, our strategy is to actively avoid any known AIS target, regardless of the type of vessel and its possible change of behavior to give the way.

The rest of the paper is organized as follows. Section 2 reviews some efforts towards the automation of the application of COLREGS for autonomous ships. Section 3 includes a brief description of the AIS system and references to softwaredefined radio projects implementing AIS receivers. The proposed strategies for defensive collision avoidance are discussed in section 4 and the paper is closed in section 5 with the concluding remarks and proposals for future directions.

\section{Automating COLREGS for robotic surface vessels}

It is clear that the current technology status of various perception systems allow ASVs to build a clear situational picture of its surroundings and provide to the computing system driving it the necessary information for avoiding collisions with other ships [3]. To be accepted in environments shared with conventional manned crafts they cannot be considered a potential danger for people or property. Many works have been proposing various techniques for implementing the rules stated in COLREGS without direct human intervention. However, the interpretation of these rules is not strict and in many cases the common sense of captains is crucial to assess practical situations and avoid collisions.

As discussed in [1], the COLREGS were designed for being used by humans and contain ambiguities that must be interpreted with a certain degree of flexibility. In that work the authors employ a behavior-based control architecture to coordinate be- 
haviors that lead to a flexible application of the rules, using an interval programming procedure for multi-objective optimization.

Other techniques exploit Genetic Algorithms (GAs) to generate near optimal COLREGs-compliant escape maneuvers [6], fuzzy logic theory [8], neural networks [10] or line-of-sight navigation with course biasing to deviate from detected obstacles [7].

A recent work [2] uses the concept of Velocity Obstacles to implement a realtime navigation planning system for roughly equivalent size and power motorized vessels, considering uncertainty in the speed and course of the meeting boats. As in several other works, this is focused on scenarios where the ASV navigates close to other vessels and perceive its surrounding environment with onboard sensors (in this case a stereo vision system).

Although many work has been done on adapting the human-based COLREGS to automatic unmanned surface vessels, the application of the rules by the boats involved in a potential collision route assumes that each vessel is able to observe the other and give the right of way when obliged by the rules. However, when the sizes, and consequently maneuverability, of two approaching boats are very different, the safest rule to apply for the smaller boat is to go away timely, regardless of what is written in COLREGS.

\section{The Automatic Identification System - AIS}

AIS is a ship location and tracking system mandatory to commercial ships above 300 tons under international navigation, all passenger ships and fishing ships above $16 \mathrm{~m}$ in length [5]. As the prices of the AIS equipments drop, this technology is being more and more adopted by smaller and pleasure ships. The system uses two radio channels in the maritime VHF band and transmits periodically various short messages reporting ship's data and its navigation status. The most important AIS message broadcasts the ship identification, navigational status, position, course and speed, which is sufficient for evaluating the potential risk of collision.

Although there are in the market various AIS receivers that are becoming cheaper, smaller and less power hungry, one alternative solution is the implementation of a fully software-defined AIS receiver in the sailboat's onboard computer. The recent launch of the low cost RTL2832/R820T/E4000-based universal radio receiver USB dongles has enabled the development of various software-defined radio projects capable of running in embedded low power computers like the RaspberryPi or the BeagleBone, including a complete AIS receiver and decoder chain (eg. the GNU AIS project - gnuais.sourceforge.net). AIS data is becoming affordable to

small and power constrained robotic sailing boats and is thus an important resource for mitigating the risk of collision with medium to large ships. 


\section{Strategies for defensive collision avoidance}

\subsection{Why defensive collision avoidance?}

In this work we consider situations arising from a potential risk of collision when a small sailing ASV encounters a large ship reporting AIS data. We assume the sailing ASV is receiving the AIS data (but not transmitting), or it has any other perception system capable of providing in real-time the location, speed and course information of the neighbor vessels. Although the location of the GPS antenna in the reporting ship is part of the AIS messages, we consider only the reported geographic position and the boat size is not relevant for the large minimum safe distance.

Instead of embedding in the navigation control system the mechanisms to apply the rules of priority established by COLREGS, we adopt an early defensive behavior to reduce the risk of collision and assume that the ASV will never have right-of-way with respect to the other vessels. This can be justified with the following arguments:

- To make use of a eventual right-of-way, a ASV must be clearly identified by the other vessel. In practical situations it may be difficult for a large ship to establish visual contact at a safe distance to allow the implementation of give-way maneuvers. On the other hand, a ASV will perceive (using the AIS data) the other ships much sooner and will have enough time to alter its navigation to avoid the encounter.

- Even if the crew of a large ship identifies a small sailboat in route of collision, with evidences of being unmanned and harmless to the ship, it is very unlikely they will change course to avoid the collision.

Additionally, a small sailing ASV is prone to the wind turbulence caused by the hull of a large ship. This wind shadow can extend leeward for a distance significantly longer than the length of the hull, affecting the sailing ability of the ASV to perform a last minute avoidance maneuver. It is thus desirable that the ASV should actively maneuver to maintain a safe distance from any moving ship significantly larger than the minimum to comfortably avoid a physical contact.

To keep clear of the other ships, a robotic sailing boat can, in some courses, reduce its speed by luffing the sails while keeping its course, or temporarily deviate from the risky route. Although a sailing robot can only navigate within the feasible points of sailing, we consider that if a escape route requires a temporary upwind course, that will be done by tacking along a narrow corridor with a few tens of meters wide. Also, the collision avoidance maneuver should not increase the risk of collision in the event of the other ship decided to maneuver to deviate, according to the rules stated in COLREGS. 


\subsection{Assumptions and definitions}

In this work we consider a scenario where a ASV $A$ and a large ship $S$ navigate along different but fixed courses and move with constant speeds. To keep the computational effort low and affordable to low performance microcontrollers, our approach is based on the continuous computation of the closest point of approach for both vessels $\left(X_{C P A}, Y_{C P A}\right)$, the time to reach that point $(T C P A)$ and the minimum distance between them (MDCPA), assuming that the wind speed, wind direction and sea state, which dictates the effective speed of the sailing boat along its course, will stay constant during the time interval required for the collision avoidance maneuver. For now we consider both vessels navigate through a straight line, and later extend to the case when a sailboat navigates upwind tacking periodically within a narrow corridor. Other variables not considered explicitly in this simple formulation (sea currents, the boat's kinematics and dynamics) are indirectly considered as we assume both ships are sailing in a straight line course with constant speed.

If the current positions of the vessels are $\left(X_{a}, Y_{a}\right)$ and $\left(X_{s}, Y_{S}\right)$, and $v_{x *}$ and $v_{y *}$ represent the $X, Y$ components of the velocities of the two vessels, the future distance between them, $d_{a s}(t)$ is given by:

$$
d_{a s}(t)=\sqrt{\left[\left(X_{a}+v_{x a} \cdot t\right)-\left(X_{s}+v_{x s} \cdot t\right)\right]^{2}+\left[\left(Y_{a}+v_{y a} \cdot t\right)-\left(Y_{s}+v_{y s} \cdot t\right)\right]^{2}}
$$

The time to the closest point of approach (TCPA) and the minimum distance of approach $(M D C P A)$ can then be calculated by minimizing function $d_{a s}(t)$. Defining the difference of velocities and positions along $X$ and $Y$ as $d_{v x}=v_{x a}-v_{x s}, d_{v y}=$ $v_{y a}-v_{y s}, d_{x}=X_{a}-X_{s}$ and $d_{y}=Y_{a}-Y_{s}$ we obtain:

$$
\begin{array}{r}
T C P A=-\left(d_{v x} \cdot d_{x}+d_{v y} \cdot d_{y}\right) /\left(d_{v x}^{2}+d_{v y}^{2}\right) \\
M D C P A=\sqrt{\left(d_{v x}^{2}+d_{v y}^{2}\right) \cdot t^{2}+\left(d_{v x} \cdot d_{x}+d_{v y} \cdot d_{y}\right) \cdot t+\left(d_{x}^{2}+d_{y}^{2}\right)} \\
X_{a_{C P A}}=X_{a}+v_{x a} \cdot T C P A \\
Y_{a_{C P A}}=Y_{a}+v_{y a} \cdot T C P A \\
X_{s_{C P A}}=X_{s}+v_{x s} \cdot T C P A \\
Y_{s_{C P A}}=Y_{s}+v_{y s} \cdot T C P A
\end{array}
$$

If the courses are not parallel, a positive TCPA means that the minimum distance of approach MDCPA will happen at time TCPA in the future. If TCPA is negative then the positions of the two vessels are monotonically diverging and there is no risk of collision.

When the sailboat navigates a upwind route, tacking periodically, the exact calculation of the previous parameters is far more complex as it depends on the geographic positions where the sailboat will effectively tack. When a robotic sailboat 
sail upwind for long distances in the same tack, this can be treated as if it is following a straight line. In the case an upwind navigation is constrained to a narrow corridor (tens to few hundreds of meters) we apply a conservative but simple rule: approximate the course of the sailboat to the straight line defining the side of the corridor closer to the approaching ship, and the sailboat speed equal to the average VMG observed along that route in the present conditions. As illustrated in figure 1, when the sailboat is in position $P$ it is considered in position $P_{A}\left(P_{B}\right)$ for analysing the CPA with ship A (ship B).

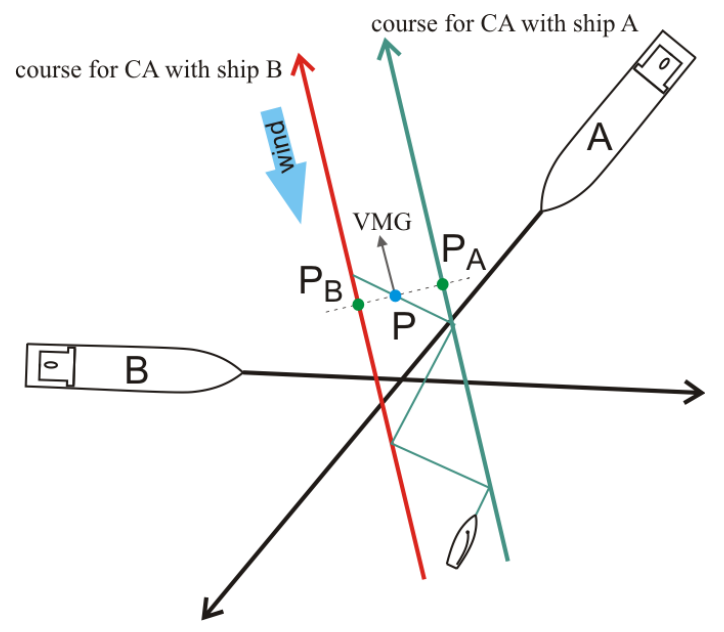

Fig. 1 Approximating the course upwind of the sailboat ASV to the limits of the upwind corridor.

\subsection{Collision avoidance maneuvers}

To reduce the risk of a collision with a ship, the robotic sailboat performs two basic maneuvers: if possible and advantageous to increase $M D C P A$, keep the course and reduce speed until MDCPA increases above the minimum acceptable; if the speed cannot be reduced while keeping the course (for example in a dead downwind course) or if slowing down will further reduce $M D C P A$, then set an escape course $C O G_{e}$ perpendicular to the ship's course, along a direction that will not cross it. While these behaviors naturally will not guarantee the avoidance of a collision, neither prove that a collision can not be avoided, they provide a very simple mechanism to mitigate practical situations when tho very dissimilar ships meet in the ocean. Besides, in this work we only address the cases when a single ship is in potential risk of collision with a ASV, what may be too restrictive under situations of dense marine traffic. 
As in COLREGS, we distinguish three different situations with respect to the relative courses of the approaching ships: heading on, overtaking and crossing routes. In the first case (heading on or opposite courses, figure 2-a), it is clear that reducing the speed of the sailboat will not contribute significantly to increase MDCPA. The same happens in the second situation (similar courses, figure 2-b: if the large ship that is overtaking the sailboat, slowing down the sailboat will increase the risk of collision; in the (unlikely) situation of being the sailing boat overtaking the ship, reducing speed will only decrease $M D C P A$ temporarily. In these cases, the strategy should be changing to the new course referred above until MDCPA measured with respect to the original course increases above the minimum. These two cases are considered when the angle difference between the absolute directions is below a certain threshold.

When the vessels are in crossing routes (figure 2-c), slowing down the sailboat will only increase MDCPA if maintaining the current speed the sailboat already guarantees a passage behind the large ship. If keeping the current route the sailboat will pass in front if the large ship, then the strategy is to sail to the escape course until the large ship has passed and MDCPA increases above the safe threshold.

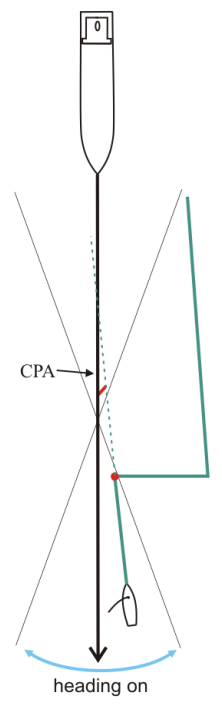

a

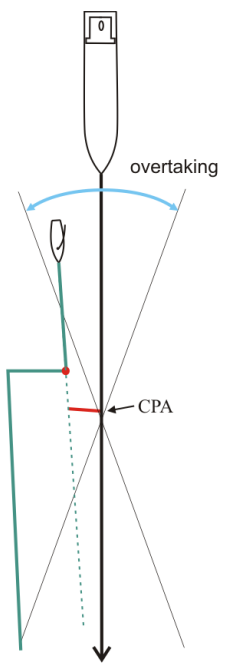

b

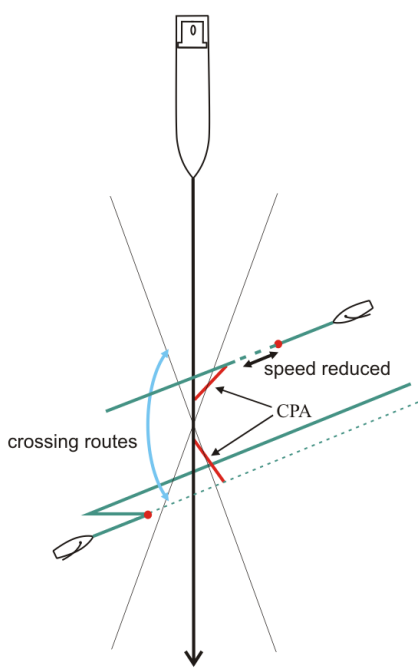

C

Fig. 2 The basic collision avoidance maneuvers.

Although slowing down can be an effective way to reduce the risk of collision in the cases illustrated above, the ability to do that will depend strongly on the type of rig and the mechanism to regulate it, and the apparent wind angle. For example, a sailboat rigging a traditional two soft sail configuration with a shrouded mast will 
not be able to slow down for large apparent wind angles, when the boom is already pushing into the shrouds.

\subsection{When to initiate a collision avoidance maneuver?}

Not too late but also not too early. A late decision will naturally compromise the efficacy of the collision avoidance maneuver and deciding too early may introduce unnecessary disturbances in the mission being accomplished by the sailboat. This time will depend on the TCPA, MDCPA and also the estimated speed of the sailboat along possible escape courses to increase the minimum distance between the vessels. The practical rule is to consider only the approaching ships when MDCPA is smaller than a minimum safe radius $M S F$ and TCPA is less than the time required to sail the distance $M S F$ along the escape route $C O G_{e}: T C P A<M S F / v_{c a}$ (where $v_{c a}$ is the minimum between the current VMG and estimated VMG of the sailboat along the escape route $C O G_{e}$ ). This guarantees that the maneuver to avoid the collision will start before crossing the route of the approaching ship.

Figure 3 illustrates two different scenarios that require sailing to a different route for avoiding a collision, showing the position where the decision to change route should occur. The sailboat navigates downwind (left) and upwind (right), the minimum safe distance $M S F$ is equal to $0.5 \mathrm{~nm}$, the downwind speed of the sailboat is $5 \mathrm{ktn}$ and the VMG upwind equal to $2 \mathrm{ktn}$. In both cases the sailboat should initiate the collision avoidance maneuver when $T C P A$ equals $15 \mathrm{~min}=0.5 / \min (2,5)=$ 0.25 hour, which guarantees enough time to navigate more than $M S F$ away from the ship route.
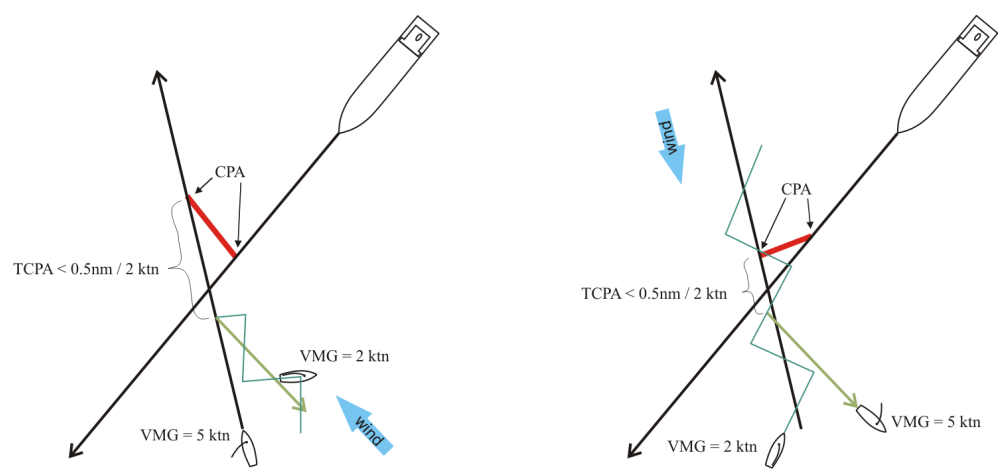

Fig. 3 Examples of points of decision for setting an escape route. 


\section{Conclusions}

Although the navigation rules established in the international regulations for preventing collisions at sea should guarantee a safe navigation for all ships adhering to them, there are many exception situations in the case of mismatch in maneuverability, size or observability of the vessels in a collision path. In long oceanic missions one real threat for small robotic sailing boats is the risk of collision with large ships that may not be able to change its route or speed for giving way, if the sailboat is identified too late or even ignored due to its small size. The basic set of rules proposed in this paper intends to establish a defensive and conservative behavior to avoid close encounters with AIS-transmitting vessels, promoting early maneuvers to reduce the risk of collision. Naturally this strategy does not make a robotic sailboat compliant with the international collision-avoidance rules, neither prevent other important hazards like collisions with small boats or entangling with floating debris for which other perception technologies are necessary. Even though this paper only considers a single ship at a time in route of collision, we will later extend the strategies presented here to take into account the identification of various ships in potential route of collision.

\section{Acknowledgements}

This work is financed by the ERDF - European Regional Development Fund through the COMPETE Programme (operational programme for competitiveness) and by National Funds through the FCT - Fundação para a Ciência e a Tecnologia (Portuguese Foundation for Science and Technology) within project "FCOMP-01-0124FEDER-037281”.

The authors are also thankful to the anonymous reviewers who provided valuable suggestions to improve the final version of the manuscript.

\section{References}

1. Benjamin, M.R., Curcio, J.A.: Colregs-based navigation of autonomous marine vehicles. Proceedings of Autonomous Underwater Vehicles (2004)

2. Kuwata, Y., Wolf, M.T., Zarzhitsky, D., Huntsberger, T.L.: Safe maritime autonomous navigation with colregs, using velocity obstacles. Oceanic Engineering, IEEE Journal of 39(1), 110-119 (2014)

3. Larson, J., Bruch, M., Ebken, J.: Autonomous navigation and obstacle avoidance for unmanned surface vehicles. In: Defense and Security Symposium, pp. 623,007-623,007. International Society for Optics and Photonics (2006)

4. International Maritime Organization: Convention on the international regulations for preventing collisions at sea (COLREGs). http://www.imo.org/About/Conventions/ListOfConventions/Pages/COLREG.aspx [accessed May 2015] (1972) 
5. United States Coast Guard, U.S. Department of Homeland Security: Automatic Identification System overview. http://www.navcen.uscg.gov/?pageName=AISmain [accessed May 2015]

6. Naeem, W., Irwin, G.W.: Evasive decision making in uninhabited maritime vehicles. In: Proceedings IFAC World Congress, Milan, Italy, August, pp. 12,833-12,838 (2008)

7. Naeem, W., Irwin, G.W., Yang, A.: Colregs-based collision avoidance strategies for unmanned surface vehicles. Mechatronics 22(6), 669-678 (2012)

8. Perera, L., Carvalho, J., Soares, C.G.: Autonomous guidance and navigation based on the colregs rules and regulations of collision avoidance. In: In Proceedings of the International Workshop Advanced Ship Design for Pollution Prevention, pp. 205-216 (2009)

9. Rynne, P.F., von Ellenrieder, K.D.: Unmanned autonomous sailing: Current status and future role in sustained ocean observations. Marine Technology Society Journal 43(1), 21-30 (2009)

10. Statheros, T., Howells, G., Maier, K.M.: Autonomous ship collision avoidance navigation concepts, technologies and techniques. Journal of navigation 61(01), 129-142 (2008) 